\title{
Prevalencia y tratamiento de mastitis en un hato lechero
}

\author{
Prevalence and treatment of bovine mastitis in a dairy \\ herd
}

Fecha recepción: 28 de Julio de 2013

Fecha Aprobación: 29 de agosto de 2013
Caroline Rosa Monteiro', Tainá Luana Vieira Lopes Zuchi², Rodrigo Antônio Pivatto ${ }^{3}$, Mário Lettieri Teixeira ${ }^{4}$

\section{Resumen}

El objetivo de este proyecto fue realizar una investigación epidemiológica de los casos de mastitis en un hato lechero, durante el período de enero de 2013 a junio de 2014. El ordeño fue mecánico, 2 veces al día. En el año 2013 se registraron 43 casos de mastitis, y 31 hasta junio de 2014. En el año 2013, el $37,21 \%$ de los casos fueron del Cuarto trasero izquierdo (CTI); 25,58\%, del Cuarto delantero derecho (CDD); 20.93\%, del Cuarto delantero izquierdo (CDI), y 16,28\%, del Cuarto trasero derecho (CTD). En el 2014, el 41,94\% de los casos fueron del CDI; el 29,03\%, del CDD; el 16,13\% del CTI, y el $12,9 \%$, del CTD. En cuanto a las drogas de uso común, en el año 2013 eran cloxacilina (24,53\% de los casos), sulfadiacina + trimetoprim (SDZ + TRIM) y gentamicina $(20,75 \%$ de los casos). En el año 2014 fueron utilizadas cloxacilina y SDZ +

\begin{abstract}
The aim of this study was to conduct an epidemiological survey in cases of mastitis in a dairy herd, from January 2013 to June 2014. Mechanical milking was done twice a day. Based on animals records, 43 cases of mastitis were recorded in 2013 compared to 31 by June 2014. In 2013, 37.21\% of the cases occurred in the left posterior teat (LPT), $25.58 \%$ in the right anterior teat (RAT), $20.93 \%$ in the left anterior teat (LAT) and $16.28 \%$ in the right posterior teat (RPT). In 2014, $41.94 \%$ of the cases were in the LAT, $29.03 \%$ in the RAT, $16.13 \%$ in the LPT and $12.9 \%$ in the RDT. Regarding the common used drugs, in 2013, cloxacillin (24.53\% of cases), sulfadiazine + trimethoprim (SDZ + TRIM) and gentamicin $(20.75 \%$ of cases) were the most used. In 2014, cloxacillin and SDZ + TRIM were used in
\end{abstract}

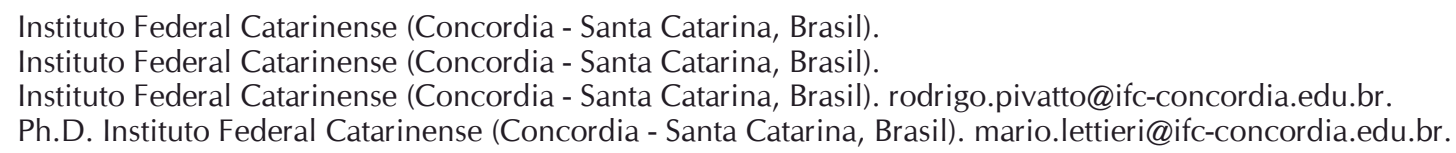


TRIM (28,57\% de los casos) y gentamicina $(22,86 \%$ de los casos). Con los resultados se puede concluir que la condición predisponente de la mastitis que afecta a las vacas es la negligencia del ordeñador en la limpieza de cuartos, junto con una mala desinfección de estos.

Palabras clave: Mastitis, Vacas, Tratamiento de la mastitis.
$28.57 \%$ of the cases, and gentamicin in $22.86 \%$. We conclude that the mastitis predisposing condition that affects the herd is the negligence of the milker cleaning and disinfecting the teats.

Keywords: Mastitis, Dairy Herd, Mastitis' Treatment. 


\section{Introducción}

La mastitis es una inflamación de la glándula mamaria que causa cambios físicos, químicos y en las propiedades organolépticas de la leche y alteraciones histopatológicas en este tejido; es una de las principales causas de pérdidas económicas, pues la cantidad y la calidad de la leche disminuyen y aumenta el costo de los medicamentos. Entre los factores predisponentes para la mastitis están la herencia, los índices zootécnicos, la etapa de la lactancia, los pastos, los traumas, las enfermedades infecciosas y el ordeño incompleto, entre otros (1).

La mastitis puede manifestarse en forma clínica o subclínica. En la forma subclínica, al contrario de la forma clínica, no se producen cambios visibles en el aspecto de la leche o en la ubre, aunque hay cambios en la composición de la leche y presencia de microorganismos patógenos. Debido a que la prevalencia de la forma subclínica es mayor que la de la clínica, las medidas para su control han recibido gran atención (2).

Aunque unos 140 microorganismos diferentes deben estar implicados en la etiología de la mastitis, Staphylococcus aureus, Streptococcus agalactiae, Streptococcus dysgalactiae, Streptococcus uberis y Escherichia coli son responsables de aproximadamente el $80 \%$ de los casos. Menos del $5 \%$ de las infecciones son causadas por Corynebacterium bovis, Pseudomonas sp., Mycobacterium sp., Nocardia sp., Aspergillus sp., Candida sp., Serratia sp. y Prototheca sp. (3).

El estado de Santa Catarina (Brasil) es el quinto productor nacional de leche, y su región oeste representa más de dos tercios de la producción estatal (4); la producción lechera en esta región se caracteriza por estar en manos de muchos pequeños propietarios, que utilizan diversos sistemas de producción con diferentes niveles tecnológicos (5). Por lo tanto, la investigación se realizó en el Instituto Federal de Santa Catarina, Campus Concordia, porque reúne factores característicos de la región lechera regional, con nivel tecnológico medio.

Los cálculos de las pérdidas económicas relacionadas con la disminución de la producción y el conocimiento de los costos son esenciales en la decisión del bovinocultor en relación con el tratamiento de la mastitis. Muchos estudios se han realizado sobre los aspectos preventivos y microbianos de esta enfermedad, pero son escasos los centrados en sus costos para la producción y el tratamiento. La escasa literatura disponible sobre la medición de los efectos económicos específicos que tienen los casos de mastitis subclínica, que estratifiquen algunos factores como la incidencia y las pérdidas económicas, la cantidad de leche desechada y la reducción de la calidad de la leche, dificultan una mejor planificación de la propiedad rural $(6,7)$.

El objetivo de este estudio fue analizar los casos de mastitis contagiosa en el hato lechero de la IFC-Concordia, en el período de enero 2013 hasta junio 2014, y develar qué factores contribuyen a ellos. Se observaron los siguientes puntos: cuáles fueron los cuartos más afectados, el tratamiento más utilizado y la repetición patológica sobre el mismo animal.

\section{Materiales y Métodos}

La investigación se llevó a cabo en un rebaño de 97 animales de la IFC-Concordia, pero fueron utilizados los datos de solo 32 animales, de la raza blanca y negro holandés y en periodo de lactancia. Los datos recogidos se refieren al período comprendido entre enero 2013 y junio 2014, que estén matriculados en los registros de cada animal.

El ordeño fue mecánico, dos veces al día, con un intervalo de 6 horas; el primero, a las 9 a.m. y el segundo, a las 3 p.m. Este procedimiento fue realizado por un solo empleado, formado por médico veterinario en el campus.

La línea de ordeño fue formada por tres grupos: primero, recién paridas en el pico de la lactancia; segundo, en la mitad o al final de la lactancia, y por último, las vacas con leche para descartar por razones de salud, especialmente por mastitis. El proceso de ordeño se inició en la etapa de predipping, que consistió en aplicar una solución basada en ácido láctico en una concentración del 3\% durante 30 segundos, y después de esto, los techos eran lavados con agua. Este proceso se repitió dos veces, con el fin de eliminar el exceso de materia orgánica. Poco después se realizó la prueba de taza de fondo negro, que consiste en recoger los primeros chorros de leche de cada cuarto y observar los cambios macroscópicos de esta, sobre todo a la presencia de grumos, sangre o 
pus. Después de la prueba, el proceso de ordeño se llevó a cabo, y al se aplicó una solución a base de ácido láctico 9\% (post-dipping).

En los casos en los que se presentaron diagnósticos de mastitis, la médico veterinaria realizó los procedimientos indicados, tales como la segregación del animal del resto y el inicio del tratamiento con medicamentos. El protocolo consistió en la aplicación de antibióticos de amplio espectro y, si era necesario, uso de medicamentos para una mayor potencia. La vía de administración preferida fue la intravenosa. En este intervalo de tiempo también se realizaron otras pruebas para confirmar el diagnóstico, como la California Mastitis Test (CMT), para la identificación de la mastitis subclínica (cada 30 días); además de la prueba de laboratorio de células somáticas (CC), recuento total de bacterias, grasas, proteínas, lactosa, que se realiza cada 40 días por el laboratorio de calidad de leche de la Compañía Integrado de Desarrollo
Agrícola de Santa Catarina (CIDASC), acreditado ante el Ministerio de Agricultura, Ganadería y Abastecimiento (MAPA).

El análisis de los datos de las historias clínicas fue adelantado con el software estadístico Stats D + (versión 1.0), utilizando el análisis estadístico descriptivo.

\section{Resultados y Discusión}

Con base en los registros de diagnóstico de la mastitis, en 2013 hubo 43 casos de mastitis, y 31 hasta junio de 2014. En el 2013, el 37,21\% de los casos fueron en el cuarto trasero izquierdo (CTI), el $25,58 \%$ en el cuarto delantero derecho (CDD), el $20.93 \%$ en el cuarto delantero izquierdo (CDI) y $16,28 \%$ en el cuarto trasero derecho (CTD). Para el año 2014, el 41,94\% de los casos fueron en el CDI, el $29,03 \%$ en el CDD, el 16,13\% en el CTI y el $12,9 \%$ en el CTD (Figura 1 ).

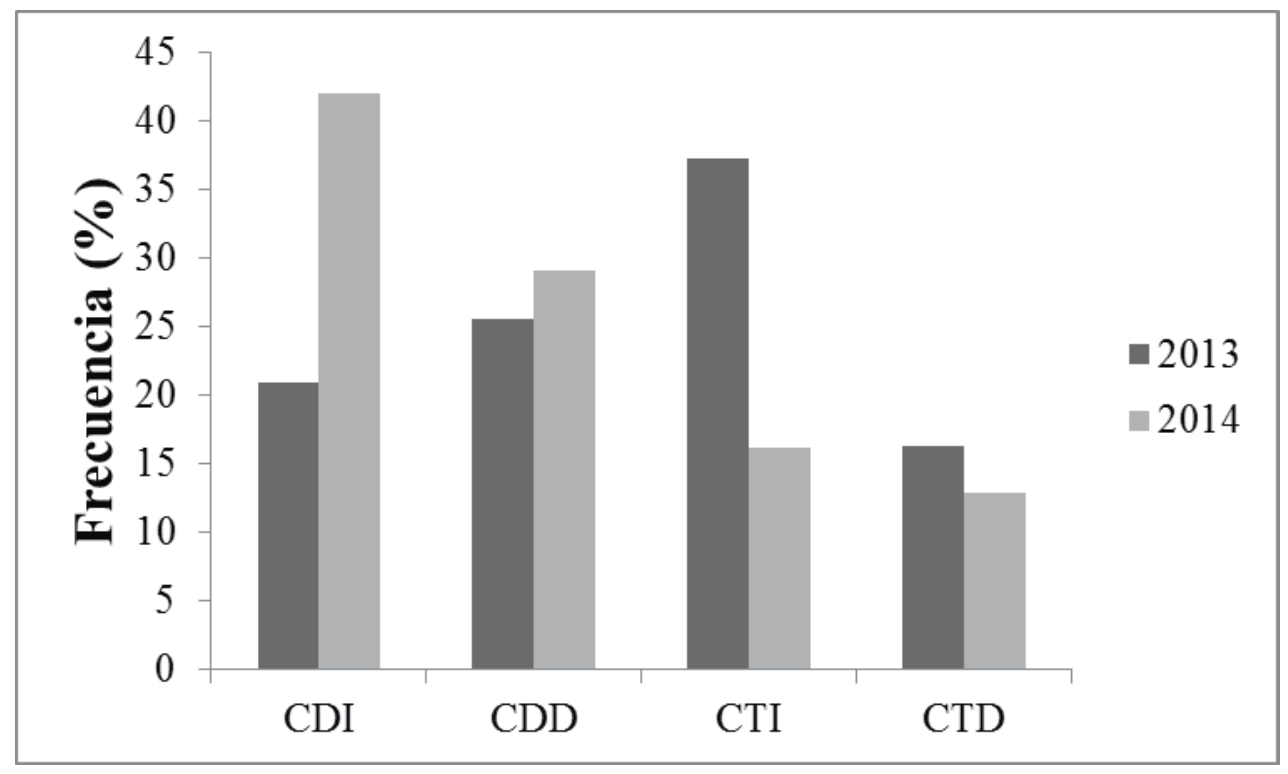

Figura 1. Frecuencia para los casos de mastitis desde enero 2013 hasta junio de 2014. Cuarto Delantero Izquierdo (CDI); Cuarto Delantero Derecho (CDD); Cuarto Trasero Izquierdo (CTI); Cuarto Trasero Derecho (CTD).

A través de la análisis de los tratamientos más utilizados, se constató que en el año 2013, el medicamento cloxacilina se utilizó en el 24,53\% de los casos; sulfadiazina + trimetoprima, en el $20,75 \%$, y gentamicina, también en el 20,75\% de los casos. Ya en el año 2014, se utilizó cloxacilina y sulfadiazina + trimetoprima en el $28,57 \%$ de los casos, respectivamente, y gentamicina en el $22,86 \%$ de los casos (Figura 2). Estos valores indican una preferencia de la terapia con antibióticos; algunos de los factores que contribuyen a esta situación son la licitación para la adquisición de insumos, 
el bajo costo del tratamiento y la facilidad de obtención de este.

Estos datos también muestran que hubo una reducción en el número de ingredientes activos que se utilizan en el tratamiento de la mastitis. En el año 2013, el 35\% de los casos de mastitis, aproximadamente, fueron tratados con fármacos distintos de los tres anteriores, mientras que en el 2014 este índice se redujo a aproximadamente el
$20 \%$; esto sugiere que, efectivamente, hubo una tendencia a utilizar mayor número de veces el mismo tratamiento (cloxacilina, sulfadiazina + trimetoprima y gentamicina), este hecho puede estar ligado a un aumento en el número de los casos de mastitis, debido a la selección de cepas resistentes a estos medicamentos, con lo que se fomenta un círculo vicioso en relación con el aumento de los casos de mastitis con la resistencia al tratamiento farmacológico.

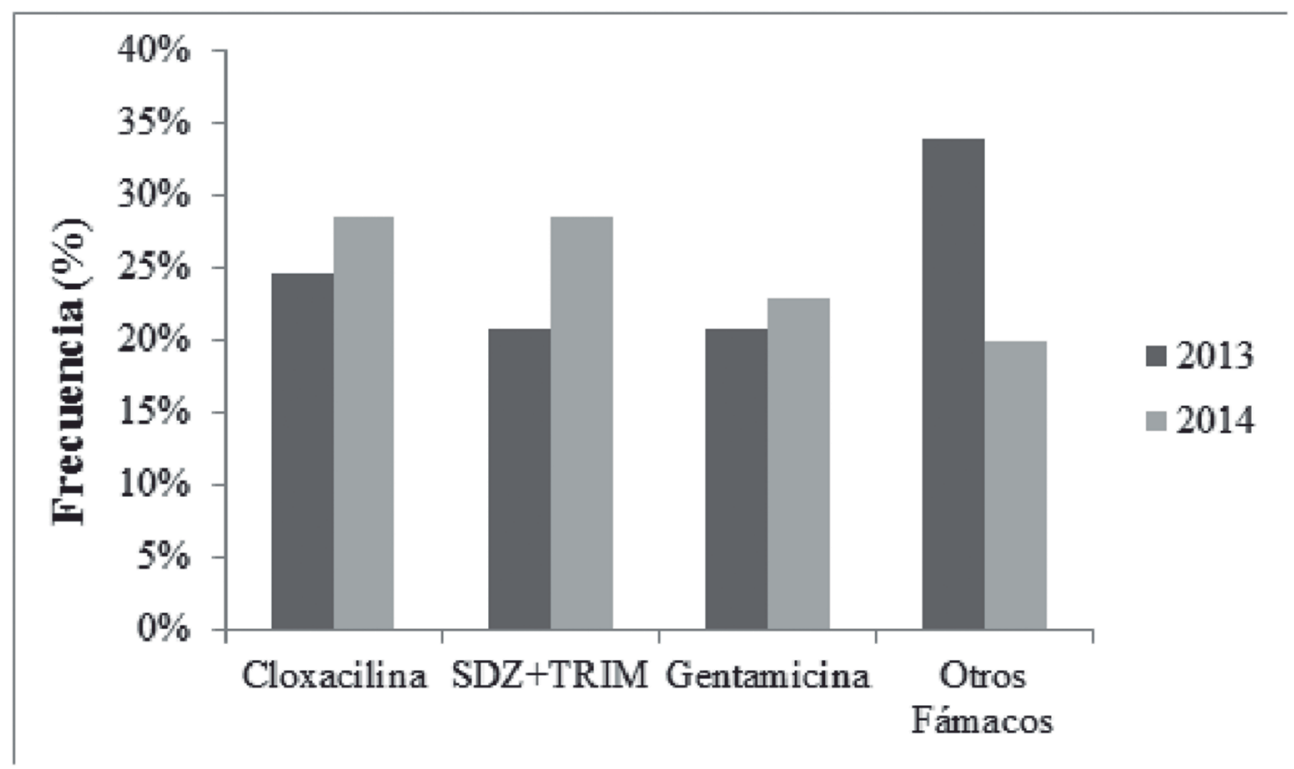

Figura 2. Frecuencia de uso de medicamentos para tratar los casos de mastitis desde enero de 2013 hasta junio de 2014. Sulfadiazina + Trimetoprima (SDZ+TRIM).

Mota y sus colegas (8) realizaron un estudio en el que se determinó que el uso inadecuado de antibióticos y desinfectantes puede promover la resistencia de las bacterias a las sustancias que se utilizan para el control de la mastitis. El desarrollo de cepas bacterianas resistentes a los antibióticos y otros medicamentos antimicrobianos es uno de los mayores problemas en el campo de la medicina. Entre los factores que explican esta característica están la genotípica, la mutación espontánea y la recombinación genética (9). Por lo tanto, estas características implican el desarrollo y la selección de cepas resistentes, que se perpetúan en el medioambiente en detrimento de cepas nativas; por lo tanto, el tratamiento inadecuado en una ubicación restringida tiene este inconveniente, facilitando el surgimiento de estos tipos de bacterias resistentes a un determinado ingrediente activo. Esta nueva situación tiene implicaciones económicas en la propiedad rural, porque el productor de leche está obligado a usar cada año nuevos fármacos para tratar los mismos animales. Sena (10), en su estudio, informó de que el alto nivel de resistencia múltiple a antibióticos puede representar un alto riesgo para la salud pública, lo que dificulta el tratamiento de enfermedades que afectan a los animales y los seres humanos, lo que agrava las condiciones médicas.

En la evaluación patológica de la recurrencia del mismo animal de la manada, se diagnosticaron 18 vacas $(56,25 \%)$ que tenían más de un caso de la mastitis durante el periodo de estudio, mientras que $5(15,63 \%)$ tenían solo uno y $9(28,12 \%)$ no desarrollaron la enfermedad durante este período. Así, de acuerdo con estos resultados, el 2014 
tiende a tener mayor número de tratamientos para la mastitis, ya que este estudio incluyó solo seis meses del año. Este gran número de vacas con recurrencia de mastitis puede sugerir un tratamiento incompleto, permitiendo la recurrencia de la enfermedad, lo que puede explicar el aumento de casos de 2013 a 2014. En este contexto, el uso de principios activos de amplio espectro y un tiempo de tratamiento reducido colaboran entre sí para producir cepas resistentes, lo que significa que hay un mayor número de repeticiones de la mastitis en animales.

Cuando se aplican correctamente las medidas profilácticas en el hato, el pre-dipping, el posdipping, la terapia de vaca seca, el tratamiento y el control de la mastitis durante la lactancia y las estrategias de eliminación, el mantenimiento adecuado de los sistemas de ordeño y las estrategias para aumentar la resistencia de la vaca, como, por ejemplo, la vacunación (11), influyen significativamente en el control de la mastitis. Además, la supervisión de un especialista, en combinación con una serie de herramientas de diagnóstico, permite evitar el empeoramiento del cuadro clínico, lo que llevaría a grandes pérdidas económicas a los bovinocultores, tales como la eliminación de la leche y hasta de los propios animales.

\section{Conclusiones}

Con estos resultados se puede concluir que el factor predisponente la mastitis que más afecta a las vacas es la negligencia del ordeñador en la limpieza de los cuartos, junto con la mala desinfección de estos.

Una de las mayores dificultades para el tratamiento eficaz de la mastitis es encontrar medios facilitadores para los exámenes bacteriológicos (antibiograma) para los cuartos afectados por la enfermedad. De esta manera, el seguimiento diario de los animales por profesionales especializados, junto con algunas herramientas de diagnóstico, contribuyen considerablemente a que la cantidad de animales afectados por la mastitis sea siempre pequeña. Por lo tanto, el bovinocultor de leche tendrá un mayor beneficio financiero de su propiedad, colaborando con el mantenimiento de la cadena productiva de esta importante actividad económica.

\section{Referencias}

(1) Bramley AJ, Cullor JS, Erskine RJ, Fox LK, Harmon RJ, Hogan JS, Nickerson SC, Oliver SP, Smith KL, Sordillo LM. Current concepts of bovine mastitis. National Mastitis Council, Madison, 1996.

(2) Troncarelli MZ, Brandão HM, Gern JC, Guimarães AS, Langoni $\mathrm{H}$. Mastite Bovina sob Nanocontrole: A Própolis Nanoestruturada como Nova Perspectiva de Tratamento para Rebanhos Leiteiros Orgânicos. Vet Zoot, 2013; 20: 124-136.

(3) Rakesh R, Swarup D, Patra R, Nandi D. Bovine Protothecal mastitis: a review. Perspect Agric Vet Sci Nut Nat Res, 2006; 1(17): 1-7.

(4) Fisher A. Produção e Produtividade de Leite do Oeste Catarinense. Race, 2011; 10(2): 337-362.

(5) Miller EA, Nesi CN. Prevalência de Agentes Causadores de Mastite, Qualidade do Leite e Conformidade com a Instrução Normativa 51. Unoesc \& Ciência - ACET, 2012; 3(2): 195-204.

(6) Hogeveen H. Mastitis is an economic problem. Proceedings of the British Mastitis Conference, Warwickshire, UK, 2005.

(7) Selvaraju G, Geetha M, Saravanan S, Manicavasaka DA. Evaluation of indirect tests for screening subclinical mastitis in dairy cows. Indian J Dairy Sci, 2013; 66(1): 55-57.

(8) Mota RA, Medeiros ES, Santos MV, Júnior JWP, Moura APBL, Coutinho LC. A etiologia das mastites em bovinos leiteiros no estado de Pernambuco (Brasil). Ci Anim Bras, 2012; 13(1): 124-130.

(9) Mota RA, Silva KPC, Freitas MFL, Porto WJN, Silva $\angle B G$. Utilização indiscriminada de antimicrobianos e sua contribuição a multirresitência bacteriana. Braz J Vet Res An Sci, 2005; 42(6): 465-470.

(10)Sena MJ. Perfil epidemiológico, resistência a antibióticos e aos conservantes nisina e sistema lactoperoxidase de Staphylococcus sp. isolados de queijos coalho comercializados em Recife-PE. 2000. 75 p Tese (Doutorado) - Escola Veterinária, Universidade Federal de Minas Gerais, Belo Horizonte, 2000.

(11)Silva MVM, Nogueira JL. Mastite: Controle e Profilaxia no Rebanho Bovino. Rev Cient Elet Med Vet, 2010; 15: 3-16. 